\title{
Facoemulsificação e ciclofotocoagulação endoscópica como tratamento primário para catarata e glaucoma coexistentes
}

\author{
Phacoemulsification and endoscopic cyclophotocoagulation as primary surgical \\ procedure in coexisting cataract and glaucoma
}

Francisco Eduardo Lopes Limaª ${ }^{1}$, Durval Moraes de Carvalho², Marcos Pereira de Ávila ${ }^{3}$

\section{RESUMO}

Objetivo: Avaliar a eficácia e segurança da facoemulsificação associada à ciclofotocoagulação endoscópica (CFE) no tratamento cirúrgico primário para catarata e glaucoma coexistentes.

Métodos: Trezentos e sessenta e oito olhos de 243 pacientes portadores de glaucoma primário de ângulo aberto (GPAA) e catarata foram incluídos no estudo. Todos os pacientes submetidos à facoemulsificação e CFE, no mesmo ato cirúrgico, no Centro Brasileiro de Cirurgia de Olhos, entre outubro de 1998 e dezembro de 2006 com, no mínimo, dois anos de seguimento, foram retrospectivamente avaliados. As cirurgias foram realizadas por um único cirurgião (FEL). Os pacientes eram excluídos se apresentassem história de qualquer cirurgia ocular ou laserterapia para o glaucoma, prévia ao procedimento. Sucesso qualificado foi definido como $5 \mathrm{mmHg}<$ pressão intraocular (Pio) $<21 \mathrm{mmHg}$ com ou sem medicações antiglaucomatosas tópicas, e sucesso total como $5 \mathrm{mmHg}$ $<$ Pio $<21 \mathrm{mmHg}$ sem o uso de medicações antiglaucomatosas em todas as visitas de acompanhamento. A necessidade de outras intervenções antiglaucomatosas durante qualquer período do acompanhamento caracterizava falência. Resultados: $O$ período médio de seguimento foi de $35,15 \pm 8,14$ meses. A Pio prévia $(23,07 \pm 5,52 \mathrm{mmHg})$ foi significativamente maior que no primeiro pósoperatório $(13,14 \pm 6,09 \mathrm{mmHg})$, e meses $1(11,03 \pm 2,59 \mathrm{mmHg}), 6(12,33 \pm$ $3,01 \mathrm{mmHg}, 12(12,19 \pm 2,19 \mathrm{mmHg}), 24(12,14+2,89 \mathrm{mmHg})$ e na última consulta $(12,29 \pm 2,44 \mathrm{mmHg})(\mathrm{p}<0,001 \mathrm{em}$ todas as ocasiões). O número de medicações pré-operatórias $(1,44 \pm 0,97)$ foi estatisticamente superior ao número de medicações na última consulta $(0,37 \pm 0,74)(p<0,001)$. Houve melhora significativa na acuidade visual ao final do seguimento $(p=0,01)$. Sucesso qualificado foi obtido em $334(90,76 \%)$ olhos, e sucesso total em $205(55,7 \%)$ dos 368 olhos operados. Observou-se como complicações Pio > 40 mmHg no pós-operatório imediato em 14,4\% (53/368) dos olhos, exsudato inflamatório na câmara anterior em 7,06\% (26/368), edema macula cistóide em 4,34\% (16/368), hipotonia transitória em 2,17\% (8/368), e íris bombé em 1,08\% (4/368) dos olhos.

Conclusão: Facoemulsificação associada à ciclofotocoagulação endoscópica é uma alternativa eficaz e segura, a médio prazo, para o tratamento de catarata e glaucoma coexistentes.

Descritores: Glaucoma/cirurgia; Endoscopia; Pressão intraocular; Extração de catarata; Avaliação de eficácia-efetividade de intervenções; Estudos retrospectivos

\section{ABSTRACT}

Purpose: To evaluate the safety and efficacy of phacoemulsification and endoscopic cyclophotocoagulation (ECP) as a primary surgical treatment for glaucoma and cataract.

Methods: Three hundred and sixty-eight eyes from 243 patients with primary open-angle glaucoma and cataract from the Centro Brasileiro de Cirurgia de Olhos that underwent an uncomplicated surgery from October, 1998 to December, 2006 with at least 2 years of follow-up were retrospectively enrolled. The patients were excluded if presented a previous ocular history of any intraocular surgery or glaucoma laser treatment. Qualified success was defined as $5 \mathrm{mmHg}<$ intraocular pressure (IOP) $<21 \mathrm{mmHg}$ with or without topical antiglaucomatous drugs, and complete success as the same IOP levels without therapy at all timepoints. Additionally, the requirement of any further glaucoma surgery was defined as failure.

Results: The mean follow-up was $35.15 \pm 8.14$ months. The preoperative IOP $(23.07 \pm 5.52 \mathrm{mmHg})$ was significantly greater than in the first postoperative day $(13.14+6.09 \mathrm{mmHg})$ and months $1(11.03+2.59 \mathrm{mmHg}), 6(12.33 \pm$ $3.01 \mathrm{mmHg}), 12(12.19 \pm 2.19 \mathrm{mmHg}), 24(12.14 \pm 2.89 \mathrm{mmHg})$ and in the last appointment $(12.29 \pm 2.44 \mathrm{mmHg})(p<0.001$ in all timepoints). The number of preoperative medications (1.44 \pm 0.97$)$ decreased $(0.37 \pm 0.74)(p<0.001)$. Furthermore, there was a significant improvement in the logMAR visual acuity $(p=0.01)$. Three hundred and thirty-foureyes (90.76\%) achieved qualified success, and 205 (55.7\%) complete success. Complications included immediate postoperative IOP spike $14.4 \%$ (53/368), postoperative fibrin exudates in anterior chamber $7.06 \%$ (26/368), cystoid macular edema 4.34\% (16/368), transitory hypotony $2.17 \%$ (8/368), iris bombé $1.08 \%$ (4/368).

Conclusion: Phacoemulsification associated with endoscopic cyclophotocoagulation is safe and effective as a primary procedure for combined glaucoma and cataract.

Keywords: Glaucoma/surgery; Endoscopy; Intraocular pressure; Cataract extraction; Evaluation of the efficacy-effectiveness of interventions; Retrospective studies
Trabalho realizado no Centro Brasileiros de Cirurgia de Olhos - CBCO - Goiânia (GO). ${ }^{1}$ Médico, Centro de Referência em Oftalmologia, Universidade Federal de Goiás - UFG -

Goiânia (GO), Brasil.
2 Médico, Centro Brasileiro de Cirurgia de Olhos, Goiânia (GO), Brasil.

3 Médico, Departamento de Oftalmologia, Centro de Referência em Oftalmologia, Universidade Federal de Goiás - UFG - Goiânia (GO), Brasil.

Endereço para correspondência: Francisco Eduardo Lopes Lima. Avenida T-2, 401, Bairro Bueno - Goiânia (GO) - CEP 74210-010 - E-mail: flimacbco@uol.com.br

Os autores não apresentam qualquer tipo de interesse comercial nos produtos ou equipamentos mencionados no texto.

Recebido para publicação em 14.06.2010

Aprovação em 01.08.2010

Nota Editorial: Depois de concluída a análise do artigo sob sigilo editorial e com a anuência do Dr. Roberto Pedrosa Galvão Filho sobre a divulgação de seu nome como revisor, agradecemos sua participação neste processo.

\section{INTRODUÇÃO}

tratamento cirúrgico da catarata e glaucoma coexistentes é controverso. A facoemulsificação atual associada à diminuição na incisão, à menor inflamação pós-operatória, e a resultados anatômico-visuais mais precoces, é hoje a técnica de escolha nas cirurgias de catarata. A trabeculectomia concomitante ainda é a opção de cirurgia antiglaucomatosa mais realizada nesses casos. O resultado desta técnica cirúrgica e suas variações (uma ou duas incisões) ou o uso per-operatório de antimetabólitos é bem conhecido ${ }^{(1-4)}$. Devido ao índice de complicações, novas técnicas cirúrgicas têm se apresentado como alternativas à faco-trabeculectomia, dentre estas a trabeculectomia isolada ${ }^{(5)}$, as cirurgias não penetrantes e as próteses para drenagem passiva em casos selecionados ${ }^{(6-7)}$. 
Recentemente, foi disponibilizado um novo instrumento para cirurgia ocular endoscópica que além do endoscópio, possibilita o tratamento de estruturas oculares com laser ${ }^{(8)}$. Apresenta a peculiar capacidade de tratar o corpo ciliar diretamente, não danificando as estruturas adjacentes. O procedimento, ciclofotocoagulação endoscópica (CFE), apresenta boa eficácia e tolerabilidade no tratamento de glaucomas refratários ${ }^{(9-10)}$ e em procedimentos cirúrgicos combinados (facoemulsificação e CFE) quando comparada à trabeculectomia ${ }^{(11-12)}$.

Esse estudo foi desenvolvido para avaliar a segurança e a eficácia, a médio prazo, de CFE associada a facoemulsificação.

\section{MÉTODOS}

Todos os pacientes submetidos, no mesmo tempo cirúrgiCo, à facoemulsificação e CFE, como tratamento cirúrgico primário para glaucoma primário de ângulo aberto (GPAA) e catarata com, no mínimo, 2 anos de seguimento, entre outubro de 1998 e dezembro de 2006, no Centro Brasileiro de Cirurgia de Olhos (CBCO), foram retrospectivamente avaliados. Todos os pacientes eram portadores de GPAA com alterações típicas do disco óptico e repercussão correspondente na perimetria computadorizada Humphrey estratégia 24-2 "full-threshold", de acordo com os critérios propostos por Hodapp-Parrish-Anderson (13). Os pacientes foram excluídos se apresentassem história de qualquer cirurgia intraocular ou laserterapia para o glaucoma prévia ao procedimento, ou complicações intraoperatórias inerentes à cirurgia de catarata (como ruptura de cápsula posterior e perda vítrea) e ainda aqueles sem seguimento pós-operatório mínimo de dois anos. De acordo com o protocolo previamente aprovado pelo comitê de ética do CBCO, todos os pacientes assinaram o Termo de Consentimento Esclarecido e foram submetidos a exame oftalmológico geral pré-operatório que constou da anotação de dados demográficos, da acuidade visual em logMAR ${ }^{(14)}$, tonometria de aplanação com tonômetro de Goldmann, biomicroscopia de segmento anterior, gonioscopia dinâmica com lente de quatro espelhos de Susmann, biomicroscopia de fundo com lente de 78 dioptrias e oftalmoscopia indireta sob midríase medicamentosa. O número de medicações (associações de drogas tópicas foram consideradas como 2) pré-operatórios, no primeiro dia, e aos 1, 6, 12,24, 36 meses e na última consulta foram anotados. Ainda foram avaliados nas visitas de seguimento acuidade visual, biomicroscopia de segmento anterior e posterior, tonometria de aplanação com tonômetro de Goldmann, gonioscopia dinâmica, bem como eventuais complicações. Sucesso qualificado foi definido como Pio entre $5 \mathrm{mmHg}$ e $21 \mathrm{mmHg}$ com ou sem medicações antiglaucomatosas tópicas, e sucesso total como Pio entre $5 \mathrm{mmHg}$ e $21 \mathrm{mmHg}$, sem a utilização de medicamentos antiglaucomatosos em qualquer uma das visitas durante o estudo. Foi considerada hipotonia transitória Pio inferior a $6 \mathrm{mmHg}$ por tempo superior a 60 dias e inferior a 6 meses.

Todas as cirurgias foram realizadas por um único cirurgião (FEL). A facoemulsificação foi realizada através de incisão em córnea clara, na região temporal superior, de cerca de 2,8 milímetros $(\mathrm{mm})$ e paracentese na região nasal superior. Após a facoemulsificação do núcleo e aspiração das massas, realizouse uma segunda paracentese, de cerca de 1,5 mm, às 9 horas no olho direito e às 3 horas no olho esquerdo. Injetou-se viscoelástico (Celoftal, ALCON, USA) entre o saco capsular e a íris e no interior do saco capsular. Introduziu-se o endoscópio (comercialmente disponível; MicroProbe, ENDOOPTIKS, Little Silver, NJ, USA) na câmara anterior por uma das paracenteses, e dirigiu-se o endoscópio para o interior do saco capsular em direção ao corpo ciliar. Após a identificação e focalização dos processos ciliares, a ciclofotocoagulação endoscópica (CFE) foi realizada em aproximadamente $210^{\circ}$ contínuos do corpo ciliar, incluin- do o terço anterior da pars plana. Durante a CFE, realizou-se depressão escleral com pinça de ponto angulada, para facilitar a exposição dos processos ciliares, o espaço entre eles e o terço anterior da pars plana e, ainda, demarcar a área a ser tratada. No olho direito, fotocoagulou-se das 3 às 10 horas e, no olho esquerdo, das 2 às 9 horas. O laser de diodo foi aplicado em modo contínuo, potência de $0,5 \mathrm{~W}$, até produzir tanto encoIhimento como mudança da coloração dos processos ciliares para branco. Em seguida, a lente intraocular dobrável de acrílico era implantada. Rotineiramente, injetou-se 0,1 mililitros $(\mathrm{ml})$ de dexametasona na câmara anterior, e $1 \mathrm{ml}$ de corticóide de depósito em espaço subtenoniano. Colírios de tropicamida $1 \%$ e a associação de dexametasona e ciprofloxacino foram instilados antes da oclusão com pomada oftálmica.

A análise estatística foi realizada através do software SPSS, versão 10,1 (SPSS Inc, Chicago, IL). O teste de Komogorov-Smirnov foi utilizado para verificar a normalidade na distribuição das amostras. Para comparar dados contínuos foi realizado o Teste T de Student Pareado, e o Teste de Wilcoxon quando as amostras apresentaram distribuição não-Gaussiana. A curva de Kaplan-Meier foi utilizada para se obter tempo de sucesso total dos olhos submetidos ao procedimento cirúrgico. O erro alfa foi corrigido de acordo com o método proposto por Cross e Chaffin (15), Porque todos os valores de $\mathrm{p}$ foram representativos, $p<0,05$ foi considerado como significativo.

\section{RESULTADOS}

Trezentos e sessenta e oito olhos (188 olhos direito e 180 olhos esquerdo) de 243 pacientes ( 87 homens e 156 mulheres) foram incluídos no estudo. A idade média dos pacientes na época da cirurgia foi de 75,8 $\pm 7,7$ anos, e o período médio de seguimento de $35,15 \pm 8,14$ meses (variando de 25 a 49 meses).

A indicação para cirurgia foi dividida em três categorias: 1. Redução da acuidade visual secundária a catarata e glaucoma controlado com medicação $(60,86 \%)$; 2 . Progressão do glaucoma determinada por piora reprodutível das alterações de campo visual (24,45\%); 3. Necessidade e/ou intolerância ao uso sistêmico de acetazolamida (14,67\%).

O comportamento da Pio e do número de medicações em uso estão ilustrados na tabela 1 e na figura 1. Sucesso qualificado foi obtido em $334(90,76 \%)$ olhos, e sucesso total em 205 $(55,7 \%)$ olhos. O gráfico de Kaplan-Meier (Figura 2) estimou o período de sucesso total em 35,67 +4,14 meses (limitado a 44 meses; intervalo de confiança: 26,32, 40,04 meses). A acuidade visual em logMAR ao final do seguimento $(0,4+0,6)$ foi significativamente melhor que pré-operatoriamente $(0,6 \pm 0,6, p=0,01)$.

Observou-se como complicações Pio $>40 \mathrm{mmHg}$ no pósoperatório imediato em 14,4\% (53/368) dos olhos quando da retirada do curativo oclusivo 3 horas após o término da cirugia. Estes olhos foram submetidos à drenagem da câmara anterior na lâmpada de fenda. Exsudato inflamatório organizado na câmara anterior ocorreu em 7,06\% (26/368), sendo que 4,34\% (16/368) evoluíram com edema macular cistóide (EMC) e baixa da acuidade visual (BAV). Apesar do tratamento para EMC, $4(1,08 \%)$ olhos ainda apresentavam BAV na última visita. Hipotonia transitória foi observada em 2,17\% (8/368) dos olhos. Estes olhos foram mantidos sob instilação de colírio de dexametasona 1\%, 4 vezes ao dia, até normalização da Pio, que ocorreu em todos estes 8 olhos antes de 6 meses de pós-operatório. Íris bombé, como consequência da formação de sinéquias posteriores com a lente intraocular, foi observada em 1,08\% (4/368) dos olhos, sendo que todos foram submetidos à iridectomia com Nd: YAG laser e evoluíram com normalização da configuração iriana. 
Tabela 1. Comportamento da pressão intraocular e número de medicações antiglaucomatosas durante o estudo. Comparação com valores pré-operatórios

\begin{tabular}{lccccccc}
\hline & Prévia & $\mathbf{1}$ dia & $\mathbf{1}$ mês & $\mathbf{6}$ meses & $\mathbf{1}$ ano & $\mathbf{2}$ anos & Última consulta \\
\hline Pio $(\mathrm{mmHg})$ & $23,07 \pm 5,52$ & $13,14 \pm 6,09$ & $11,03 \pm 2,59$ & $12,33 \pm 3,01$ & $12,19 \pm 2,19$ & $12,14 \pm 2,89$ \\
$\mathrm{P}^{*}$ & - & $<0,001$ & $<0,001$ & $<0,001$ & $<, 0,001$ & $<0,001$ \\
Medicações & $1,44 \pm 0,97$ & $0,0 \pm 0,0$ & $0,18 \pm 0,48$ & $0,25 \pm 0,59$ & $0,38 \pm 0,69$ & $0,37 \pm 0,68$ & $<, 44$ \\
$\mathrm{P}^{\#}$ & - & $<0,001$ & $<0,001$ & $<0,001$ & $<0,001$ & $<0,001$ & $<01$ \\
\hline
\end{tabular}

${ }^{*}=$ teste $T$ de student pareado

\#=teste de Wilcoxon

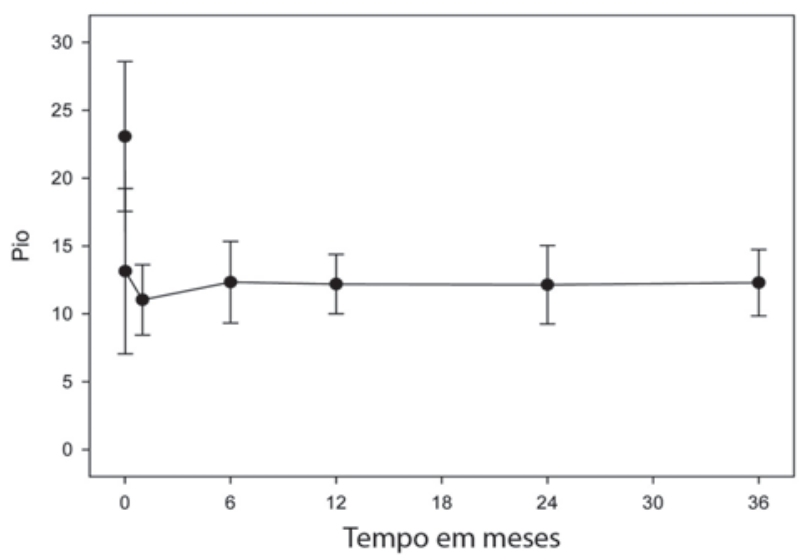

Figura 1. Variação da pressão intraocular pós-operatória.

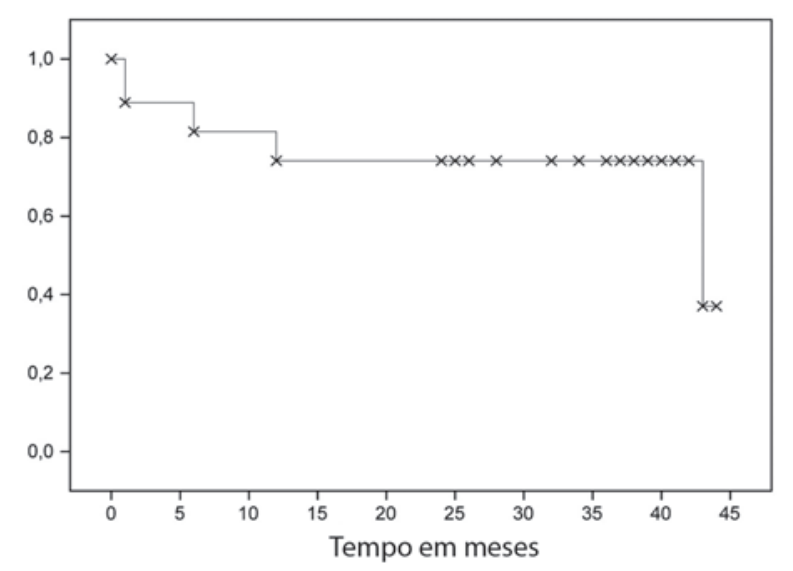

Figura 2. Curva de sobrevivência de Kaplan-Meier.

\section{DISCUSSÃO}

A trabeculectomia associada à facoemulsificação geralmente apresenta índices de sucesso satisfatórios em relação ao controle pressórico a longo prazo, principalmente se combinada com o uso de antimetabólitos ${ }^{(1)}$. Entretanto, devido ao índice acumulativo de complicações com a cirurgia fistulizante ${ }^{(16-17)}$, novos procedimentos antiglaucomatosos têm sido propostos.

Neste estudo, a Pio foi significantemente reduzida após a facoemulsificação associada a CFE (Tabela 1), com poucas complicações. Nota-se, que o controle da Pio já acontece no primeiro dia pós-operatório, e perdura de forma linear (constante) ao longo de todo o acompanhamento (Figura 1, média de 36 meses).
Em um estudo prospectivo, randomizado, Gayton et al. ${ }^{(11)}$ compararam a eficácia e segurança entre faco-trabeculectomia e faco-CFE. No grupo faco-CFE, 240-270 graus dos processos ciliares foram fotocoagulados. Com tempo de seguimento médio de 2 anos, o índice de sucesso em obter Pio $<19 \mathrm{mmHg}$ sem medicação foi $42 \%$ no grupo da faco-trabeculectomia e $30 \%$ no grupo da faco-CFE. Observa-se que $90,76 \%$ dos olhos neste estudo atingiram Pio $<21 \mathrm{mmHg}$ com um número mínimo de medicações (média inferior a uma medicação, Tabela 1) e que $55,7 \%$ dos olhos operados não necessitaram de medicação em nenhum momento do seguimento. Importante salientar que no estudo conduzido por Gayton et al. ${ }^{(11)}$ não foi realizado depressão escleral para melhor expor o espaço entre as cristas ciliares e também não foi tratado o terço anterior da pars plana como descrito no presente estudo. Estes fatos provavelmente estão relacionados ao índice de sucesso inferior descrito por aqueles autores em relação ao presente estudo.

Apesar do sucesso ter sido definido como Pio $<21 \mathrm{mmHg}$, a média foi inferior, em torno de $12 \mathrm{mmHg}$, níveis, segundo a literatura, suficientes para se evitar ou retardar a perda de campo visual inclusive em pacientes com glaucoma avançado(18).

As complicações mais temidas com os procedimentos ciclodestrutivos são a redução da acuidade visual e a atrofia do globo ocular (phthisis bulbi) ${ }^{(12,19)}$. Com o advento da CFE, o índice de phthisis bulbi foi reduzido drasticamente ${ }^{(0,20)}$, não tendo sido relatado em pacientes submetidos à cirurgia primária(11).

Observou-se, no presente estudo, hipotonia transitória em 2,17\% (8/368) dos olhos. Todos estes olhos evoluíram com normalização da Pio entre 3 e 6 meses de pós-operatório apenas com o uso de corticóide tópico. Estudos sobre CFE em glaucomas refratários ${ }^{(8-10,20)}$ relataram índices de hipotonia superior a 6 meses entre 0\% e 20\%, e índices de phthisis bulbi entre 0\% e 5\%, ambas complicações mais frequentes em pacientes portadores de glaucoma neovascular.

Concluindo, facoemulsificação e CFE é uma alternativa segura e eficaz no controle pressórico, a médio prazo, como tratamento cirúrgico primário em pacientes com catarata e GPAA coexistentes. Estudos prospectivos, comparativos, randomizados são necessários para estabelecer o papel da facoemulsificação associada a CFE em relação à faco-trabeculectomia.

\section{REFERÊNCIAS}

1. Jampel HD, Friedman DS, Lubomski LH, Kempen JH, Quigley H, Congdon N, et al. Effect of technique on intraocular pressure after combined cataract and glaucoma surgery: An evidence-based review. Ophthalmology. 2002;109(12):2215-24; quiz $2225,2231$.

2. Friedman DS, Jampel HD, Lubomski LH, Kempen JH, Quigley H, Congdon N, et al. Surgical strategies for coexisting glaucoma and cataract: an evidence-based update. Ophthalmology. 2002;109(10):1902-13. Comment in: Ophthalmology. 2004; 111(2):408-9.

3. Porges $Y$, Ophir A. Surgical outcome after early intraocular pressure elevation following combined cataract extraction and trabeculectomy. Ophthalmic Surg Lasers. 1999;30(9):727-33. 
4. Rockwood EJ, Larive B, Hahn J. Outcomes of combined cataract extraction, lens implantation, and trabeculectomy surgeries. Am J Ophthalmol. 2000;130(6):704-11. Tanihara H, Honjo M, Inatani M, Honda Y, Ogino N, Ueno S, et al. Trabeculotomy combined with phacoemulsification and implantation of an intraocular lens for the treatment of primary open-angle glaucoma and coexisting cataract. Ophthalmic Surg Lasers. 1997;28(10):810-7.

6. Gandolfi S, Traverso CF, Bron A, Sellem E, Kaplan-Messas A, Belkin M. Short-term results of a miniature draining implant for glaucoma in combined surgery with phacoemulsification. Acta Ophthalmol Scand Suppl. 2002;236:66

7. Hoffman KB, Feldman RM, Budenz DL, Gedde SJ, Chacra GA, Schiffman JC. Combined cataract extraction and Baerveldt glaucoma drainage implant: indications and outcomes. Ophthalmology. 2002:109(10):1916-20.

8. Uram M. Ophthalmic laser microendoscope ciliary process ablation in the management of neovascular glaucoma. Ophthalmology. 1992;99(12):1823-8.

9. Lima FE, Carvalho DM, Beniz J, Avila M. Ciclofotocoagulação endoscópica em glaucomas refratários. Rev Bras Oftalmol. 1997:56(6):387-93.

10. Chen J, Cohn RA, Lin SC, Cortes AE, Alvarado JA. Endoscopic photocoagulation of the ciliary body for treatment of refractory glaucomas. Am J Ophthalmol. 1997: 124(6):787-96.

11. Gayton JL, Van Der Karr M, Sanders V. Combined cataract and glaucoma surgery: trabeculectomy versus endoscopic laser cycloablation. J Cataract Refract Surg. 1999;25(9):1214-9.
12 Rebolleda G, Muñoz FJ, Murube J. Audible pops during cyclodiode procedures. Glaucoma. 1999;8(3):177-83.

13. Hodapp E, Parrish II RK, Anderson DR. Clinical decisions in glaucoma. St. Louis: Mosby: 1993.

14. Holladay JT. Proper method for calculating average visual acuity. J Refract Surg. 1997;13(4):388-91.

15. Cross EM, Chaffin WW. Use of the binomial theorem in interpreting results of multiple tests of significance. Educ Psychol Meas. 1982-42(1):25-34

16. Costa VP, Smith M, Spaeth GL, Gandham S, Markovitz B. Loss of visual acuity after trabeculectomy. Ophthalmology. 1993;100(5):599-612.

17. Bindlish R, Condon GP, Schlosser JD, D'Antonio J, Lauer KB, Lehrer R. Efficacy and safety of mitomycin-C in primary trabeculectomy: five-year follow-up. Ophthalmology. 2002;109(7):1336-41; discussion 1341-2.

8. The Advanced Glaucoma Intervention Study (AGIS): 7. The relationship between control of intraocular pressure and visual field deterioration. The AGIS Investigators. Am J Ophthalmol. 2000;130(4):429-40.

19. Shields MB. Cyclodestructive surgery for glaucoma: past, present, and future. Trans Am Ophthalmol Soc. 1985:83:285-303.

20. Lima FE, Magacho L, Canvalho DM, Susanna R Jr, Avila MP. A prospective, comparative study between endoscopic cyclophotocoagulation and the Ahmed drainage implant in refractory glaucoma. J Glaucoma. 2004;13(3):233-7.

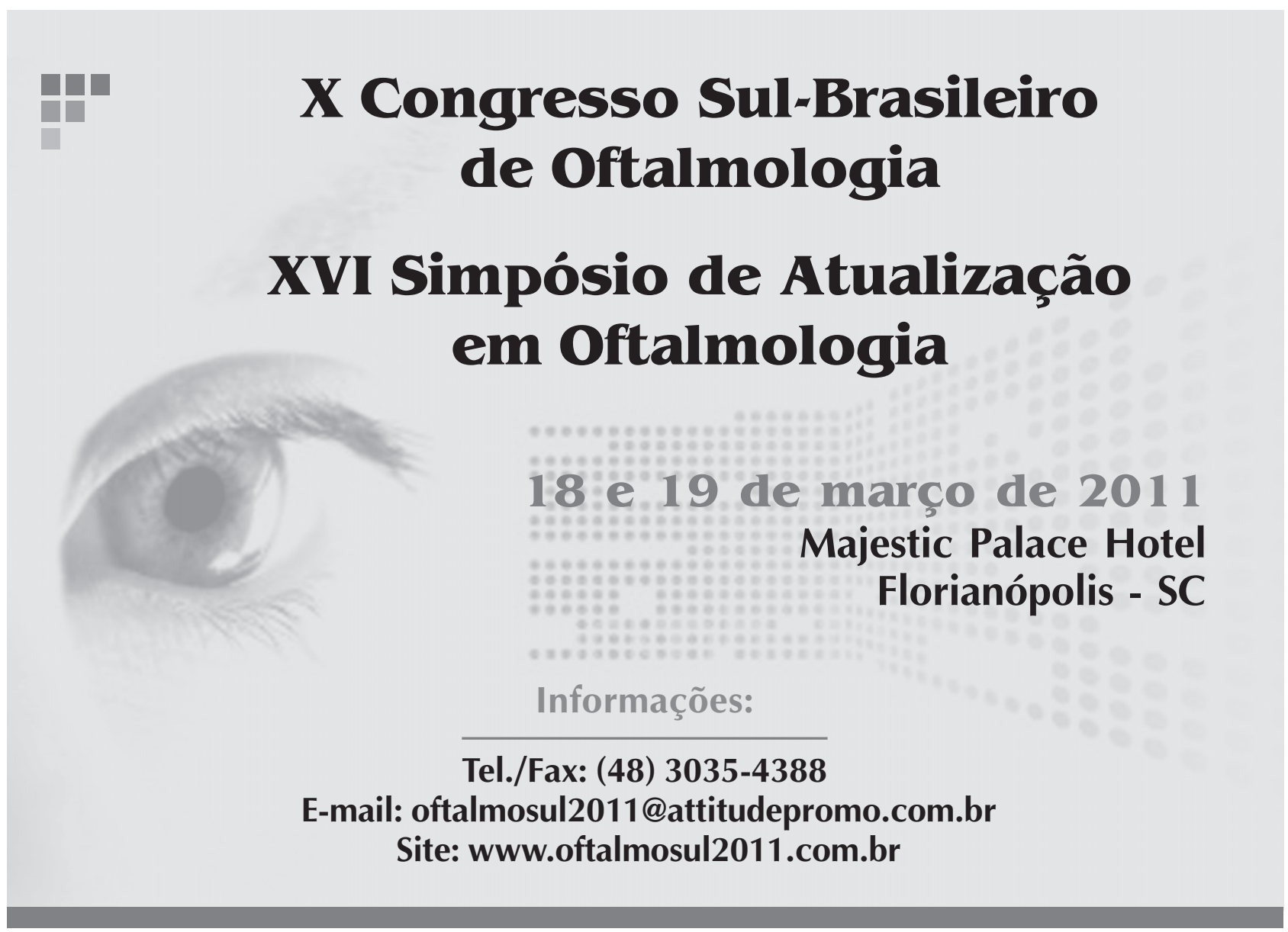

